TITLE:

\title{
Verification of criterion-related validity of the evaluation method of postural stability using the frame subtraction method.
}

\section{AUTHOR(S):}

Ota, Megumi; Tateuchi, Hiroshige; Hashiguchi, Takaya; Ogino, Yasuhiro; Ichihashi, Noriaki

\section{CITATION:}

Ota, Megumi ... [et al]. Verification of criterion-related validity of the evaluation method of postural stability using the frame subtraction method.. Journal of biomechanics 2020, 109: 109958.

\section{ISSUE DATE:}

2020-08-26

URL:

http://hdl.handle.net/2433/255859

\section{RIGHT:}

(c) 2020. This manuscript version is made available under the CC-BY-NC-ND 4.0 license

http://creativecommons.org/licenses/by-nc-nd/4.0/.; The full-text file will be made open to the public on 26 August 2021 in accordance with publisher's 'Terms and Conditions for Self-Archiving'.; この論文は出版社版でありません。引 用の際には出版社版をご確認ご利用ください。; This is not the published version. Please cite only the published version. 


\section{Title}

Verification of criterion-related validity of the evaluation method of postural stability using the frame subtraction method

\section{Author names and affiliations}

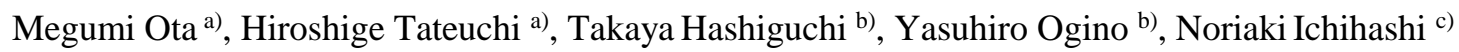

a. Department of Preventive Physical Therapy, Human Health Sciences, Graduate School of Medicine, Kyoto University,

Kyoto, Japan

b. Mixi Incorporated, Tokyo, Japan

c. Department of Physical Therapy, Human Health Sciences, Graduate School of Medicine, Kyoto University, Kyoto, Japan

\section{Corresponding author}

Ota Megumi

Department of Preventive Physical Therapy, Human Health Sciences, Graduate School of Medicine, Kyoto University,

Kyoto 606-8507, Japan

53 Kawara-cho, Shogoin, Sakyo-ku, Kyoto, Japan

Tel/Fax: +81-75-751-3948

Email: ota.megumi.7e@kyoto-u.ac.jp

\section{Key words}

Frame subtraction method; Motion analysis; Marker less system 


\begin{abstract}
It is important to quantify the postural stability. The frame subtraction method can calculate the motions of a subject, and might be easier to implement, with lower costs. However, validity of the evaluation of postural stability using this method have not been validated yet. Therefore, the purpose of this study was to verify criterion-related validity of the frame subtraction scores and the center of pressure (COP) parameters during maintenance of single leg standing.
\end{abstract}

Twenty two healthy young subjects participated in this study. Motion tasks comprised right leg standing with eyes open and closed. The total length of COP displacements (LNG), Root mean square (RMS) area, anterior - posterior (AP) range , medial - lateral (ML) range were recorded using the force plate. Simultaneously, the motion images were acquired with digital video cameras from the front and right sides. After the motion images were analyzed using the frame subtraction method, the frame subtraction scores (maximum / sum of the frame subtraction score on each plane / the frontal and sagittal planes) were measured. To confirm the validity, Spearman's rank correlation coefficient between the frame subtraction scores and the COP parameters was calculated.

The sum of the frame subtraction score on the frontal plane was significantly correlated with all COP displacements in the single leg standing. The result of this study indicated that the frame subtraction method could be applied to the evaluation of balance task with postural sway such as maintenance of single leg standing. The frame subtraction method is low cost and easy owing to its marker-less systems.

\title{
1. Introduction
}

Postural sway is one of the well-known evaluations of postural stability and is not limited to the elderly and patients with balance disorder, but is also used to assess healthy subjects and athletes. The force plates and marker - based systems such as three directional motion analysis systems and accelerometer are generally used in order to gain objective data on postural sway. Since the displacement of center of pressure (COP) during balance tasks reflects balance ability to maintain the center of mass within the base of support (Winter et al., 2003), the COP parameters are used for quantitative evaluation method of postural stability. In particular, the total length of COP displacements (LNG) and the root mean square area (RMS area) are typically used as COP parameters (Albertsen et al., 2017, Bauer et al., 2008). LNG indicates the distance of COP, and the RMS area indicates the range of COP. In the preceding study, gradual increase in the COP parameters due to age have been reported (Abrahamová et al., 2007, Prieto et al., 1996, Wingert et al., 2013). Moreover, it 
has reported that the COP parameters are the variables that distinguish older adult fallers from non-fallers (Pizzigalli et al., 2016), and postural sway might be an indicator of falls risk (Piirtola et al., 2006, Pizzigalli et al., 2016, Pua et al., 2017). Therefore, to understand changes in motor function and the risk of fall caused by aging and diseases with balance disorder objectively, it is necessary to quantify postural stability.

Conversely, there are some disadvantages associated with measurement using equipment such as force plates and marker-based systems. These conventional equipment are expensive, and require much time and technological skill for attaching sensors. Therefore, these measurement equipment are used in limited special environments such as hospitals and laboratories. In most clinical fields, since it is difficult to establish an environment where evaluations of postural stability could be performed using these measurement equipment, it is hard to say that objective evaluations are performed.

The frame subtraction method is one of the key techniques for the automatic image analysis method. The method enables the detection of targeting objects by calculating subtractions between three consecutive images (Alex et al., 2014, Ferryman et al., 2013, Singla, 2014, Guo et al., 2017, Ramya et al., 2016). It has conventionally been applied to count traffic volume on the road and the number of customers in a shop using fixed cameras. As application methods of the frame subtraction method in the medical field, it is used to detect foreign substances in an injection solution (Lu et al., 2011) or to track human sperm cells (Imani et al., 2014). In an environment where nothing moves except for the targeting subject, the motions of the targeting subject can be quantified by the frame subtraction method. By recording each subject during maintenance of single leg standing using one or two digital video cameras, it may be possible to analyze the postural sway, such as motions of the trunk and lower limbs, from the captured images using the frame subtraction method. However, there have been no studies applying the frame subtraction method to evaluation of postural stability. Therefore, we investigate whether this method can be applied to evaluate postural stability during maintenance of single leg standing.

The frame subtraction method is an Open Source Software and included in the OpenCV library (OpenCV 4.1.1). Image analysis by the frame subtraction method only requires standard digital video cameras or digital video cameraequipped tablets on the market, thus the costs could be reduced. Therefore, this system is easier and portable compared to conventional methods, and can be used outside the laboratory. If it is possible to evaluate postural stability using the frame subtraction method, this method could be more applicable in the clinical and sports field. However, there has been no attempt to confirm the correlations between the frame subtraction scores using the frame subtraction method and the COP 
parameters measured using the force plate. Thus, the criterion-related validity of the evaluation of postural stability using the frame subtraction method has not been clarified.

Therefore, the purpose of this study was to verify the criterion-related validity of the evaluation of postural stability using the frame subtraction method.

\section{Methods}

\subsection{Subjects}

The subjects were 22 healthy young people (16 males and 6 females; age, $25.9 \pm 4.6$ years; height, $167.6 \pm 7.7 \mathrm{~cm}$; mass, $60.6 \pm 10.7 \mathrm{~kg})$. Those who had a serious history, such as present or past history of ligament injury, fractures, and surgery in the extremities and trunk, were excluded. This study was approved in advance by the ethics committee of Kyoto University (approval number: R1823), and all subjects were given full written explanations before consenting to participate.

\subsection{Motion tasks}

The subjects were asked to keep standing on a single leg for $15 \mathrm{~s}$ with their hands folded across under their chest, and left hip flexed 90 degrees. Although other joints were allowed to move freely, stepping was prohibited. Eyes open conditions and eyes closed conditions were indicated randomly.

\subsection{Collection of data}

The measuring intervals of digital video cameras - equipped tablets and the force plate were synchronized. Within the range of $15 \mathrm{~s}$ of single leg standing, consecutive $10 \mathrm{~s}$ where the subjects remained stable was used for analysis. After several practice trials, these trials were recorded. The data from each trial ware calculated. Mean values from three successful trials were used for statistical analysis. The frame subtraction scores for the same image twice confirmed that the results were in complete agreement.

\subsubsection{The COP parameters}

To measure the balance ability in the conventional way, the COP parameters during maintenance of single leg 
standing were collected using a force platform (Kistler Japan Co., Ltd. Tokyo, Japan). The force platform data were sampled at a frequency of $120 \mathrm{~Hz}$ and low-pass-filtered at $10 \mathrm{~Hz}$. LNG, RMS area, range in anterior - posterior direction (AP range), range in medial - lateral direction ML range (ML range) were calculated as the COP parameters.

\subsubsection{The frame subtraction method used in this study}

The motion images were recorded using each digital video camera - equipped tablets from the front and right side of the subjects at a sampling rate of $60 \mathrm{~Hz}$. Fig 1 shows the experimental setup. First, the captured images ware processed by the frame subtraction method (Alex et al., 2014), and the frame subtraction scores on the frontal and the sagittal plane were quantified. Next, the frame subtraction scores on the frontal and the sagittal planes were summed for each frame. The maximum and sum of the frame subtraction scores for $10 \mathrm{~s}$ were calculated as an indicator of the balance ability. In an environment where nothing moves except for the targeting subject, the differences between images are caused by the motions of the subject only. Therefore, the frame subtraction scores obtained by making the differences between images into scores indicate the motions of the subject.

1. Prepare three consecutive images $\left(I_{1}, I_{2}\right.$, and $\left.I_{3}\right)$

2. Calculate the absolute value of the difference between images $I_{1}$ with $I_{2}$, and between images $I_{2}$ with $I_{3}$, and make two different images $\left(I_{d 1}\right.$ and $\left.I_{d 2}\right)$

$$
\begin{aligned}
& I_{d 1}(\mathrm{x}, \mathrm{y})=\left|I_{1}(\mathrm{x}, \mathrm{y})-I_{2}(\mathrm{x}, \mathrm{y})\right| \\
& I_{d 2}(\mathrm{x}, \mathrm{y})=\left|I_{2}(\mathrm{x}, \mathrm{y})-I_{3}(\mathrm{x}, \mathrm{y})\right|
\end{aligned}
$$

3. Calculate the logical product of two subtraction images $\left(I_{d 1}\right.$ and $\left.I_{d 2}\right)$ and create a logical product image $\left(I_{a}\right)$

$$
I_{a}(\mathrm{x}, \mathrm{y})=I_{d 1}(\mathrm{x}, \mathrm{y}) \wedge I_{d 2}(\mathrm{x}, \mathrm{y})
$$

4. Perform binarization processing on logical product image $I a$ ( $T$ is a threshold)

$$
I_{m}(\mathrm{x}, \mathrm{y})=255\left(I_{a}(\mathrm{x}, \mathrm{y})>T\right), 0\left(I_{a}(\mathrm{x}, \mathrm{y}) \leqq T\right)
$$

5. Calculate the total value for each frame and determine it as the frame subtraction score for that frame. 
To confirm the criterion-related validity, Spearman's rank correlation coefficient between the frame subtraction scores and the COP parameters was measured. First, correlations between the frame subtraction scores on the frontal plane or sagittal plane with all COP parameters were investigated. The sum of the frame subtraction scores for the frontal and sagittal planes were calculated for each frame. The scores were determined as the frame subtraction score on both planes, and were investigated for correlation with all COP parameters similarly. The level of significance was set at $\mathrm{p}<0.05$. Statistical analysis were performed using IBM SPSS Statistics, version 26 (IBM Japan Ltd., Tokyo, Japan).

\section{Results}

Mean values \pm standard deviation for the frame subtraction scores and the COP parameters were described in Table 1 and Table 2. Table 1 summarizes the results during maintenance of single leg standing with eyes open, and Table 2 summarizes the results during maintenance of single leg standing with eyes closed. Typical examples of the displacement of the COP and the frame subtraction scores on the frontal and sagittal planes in stable and unstable trials were shown in Fig 2. The scatter diagrams of the sum of the frame subtraction scores on the frontal plane and each COP parameter for single leg stance with eyes open (upper panel: a) and closed are shown in Fig 3.

As shown in Table 3, regarding single leg standing with eyes open, there were significant correlations between the maximum of the frame subtraction score on the frontal plane with ML range, as well as between the sum of the frame subtraction score on the frontal plane with LNG, RMS area, AP range, and ML range. As shown in Table 4, regarding single leg standing with eyes closed, there were significant correlations between all frame subtraction scores on the frontal, sagittal, and both planes with all COP parameters, except between the maximum frame subtraction score on the sagittal plane and the ML range.

\section{Discussions}

In this study, the frame subtraction scores were quantified from the captured images of subjects during maintenance of single leg standing recorded using two digital video cameras, and the correlations between the frame subtraction scores and the COP parameters measured using the force plate were confirmed. This study is the first attempt to apply the frame subtraction method to the evaluations of postural stability. The main findings of this study are that there were significant positive correlations among the sum of the frame subtraction score on the frontal plane and LNG, RMS 
area, AP range, and ML range, during maintenance of single leg standing with eyes open and closed.

To evaluate postural stability objectively, it is necessary to quantify the postural sway. In recent years, video analysis technologies have improved tremendously. Previous studies have attempted to apply those techniques to the objective evaluation of postural stability (Allin et al., 2008, Goffredo et al., 2006, Kawa et al., 2018). It has been reported that each body part of subjects during maintenance of stable standing can be estimated from captured images taken with one digital video camera; therefore, the center of mass (COM) trajectories (Goffredo et al., 2006) and the periods of leg up during maintenance of single leg standing (Kawa et al., 2018) were measured. However, in previous studies, validity of the evaluation of postural stability using a conventional marker-less system have not been verified. Therefore, it cannot be said at present that video analysis technologies with marker-less systems have been sufficiently studied in the evaluation of postural stability.

The primary finding of this study is that there are significant positive correlations among the sum of the frame subtraction score on the frontal plane and all COP parameters during maintenance of single leg standing with eyes open and closed. The present results suggest that the sum of frame subtraction score on the frontal plane can indicate instability during maintenance of single leg standing with eyes open and closed. The frame subtraction scores on the frontal and sagittal planes can be measured from captured images taken with digital video cameras from the front and support side easily. Because no measurement equipment or sensors are required, the evaluation of postural stability using the frame subtraction method has low cost, which favors its use in many clinical fields. However, there were differences in results during maintenance of single leg standing between eyes open and closed. Regarding single leg standing with eyes closed, all frame subtraction scores on the frontal, sagittal, and both planes correlated with all COP parameters except between the maximum frame subtraction score on the sagittal plane and the ML range. Regarding single leg standing with eyes open, the sum of the frame subtraction score on the frontal plane only correlated with all COP parameters, and there were no correlations among the frame subtraction scores on the sagittal plane and both planes and all COP parameters. The reason is considered as follows. During maintenance of single leg standing with eyes open, there may have been individual differences in the posture control pattern on the sagittal plane. Although the COP parameters are closely related to trajectories in the center of gravity, joint motions do not necessarily link with trajectories in the center of gravity. 
the center of gravity move even without remarkable joint motions such as ankle strategy. In the former case, even if the COP parameters are small, the frame subtraction scores, especially on the sagittal plane, would not be small; in the latter case, even if the COP parameters are large, the frame subtraction scores would not be large. For this reason, regarding maintenance of single leg standing with eyes open, the frame subtraction scores on the sagittal plane might have been difficult to relate to the COP parameters. Although the images on the frontal and sagittal planes were analyzed in this study, regardless of single leg standing with eyes open or closed, the frame subtraction scores on the frontal plane only were associated with all COP parameters. Thereby, when evaluating maintenance of single leg standing, it is concluded that the captured images on the frontal plane only are sufficient to analyze the evaluation of postural stability. In other words, it is possible to propose that postural sway during maintenance of single leg standing can be evaluated using one digital video camera only.

Several limitations to this study should be noted. First of all, there are limitations due to the features of the frame subtraction method. Since all moving objects are identified by the frame subtraction method, it necessary to establish one or two digital video cameras and enough space without moving objects except the subject. Another limitation is the accuracy issue. Because the images are filtered for the purpose of cutting noise, the frame subtraction method could not capture inappreciable fluctuations. Thereby, it is thought that the evaluation method of postural stability using the frame subtraction method might not be suitable for easy balance tasks without remarkable joint motions, such as static standing with eyes open in healthy subjects. However, in the evaluation method of postural stability measured using a force plate, the subjects could not be measured accurately unless they stand on the force plate directly, while the evaluation method of postural stability using the frame subtraction method could be applied even under conditions on an unstable board or with weights. Finally, there is a limitation associated with this study. In this study of healthy subjects, the AP ranges during maintenance of single leg standing had significantly positive correlations with the ML ranges ( $r=0.63-0.68, \mathrm{p}<0.01)$. Therefore, the frame subtraction scores on the frontal plane, primarily indicating postural sway on the frontal plane, might affect the MP range directly and AP range indirectly. However, it has not yet been verified that the frame subtraction method can be applied to the evaluation of postural stability with balance disorder, especially with remarkable postural sway on the sagittal plane. Further studies are needed to apply the frame subtraction method to the evaluation of postural stability in other balance tasks or other subjects. 
In conclusion, the current study verified the criterion-related validity of the frame subtraction method based on the evaluation of postural stability. In the balance task with large postural sway, such as single leg standing with eyes closed, except for between the maximum frame subtraction score on the sagittal plane and ML range, all frame subtraction scores on the frontal, sagittal, and both planes were associated with all COP parameters. In the balance task with small postural sway, such as maintenance of single leg standing with eyes open, only the sum of the frame subtraction score on the frontal plane was associated with all COP parameters. In this study, although two digital video cameras were installed ahead and to the right of the subject, regardless of the range of the postural sway, it was possible to measure postural sway with only one digital video camera on the frontal plane, and thus evaluate postural stability. Since the frame subtraction method can reduce costs and time because of its marker-less system, it may be possible to propose a new technique for the evaluation of postural stability during maintenance of single leg standing as an alternative to the use of conventional equipment such as force plates.

\section{Acknowledgements}

We thank all of our study subjects for their participation and engineers of Smart Health Incorporated. This study was supported by a joint research fund from Smart Health Incorporated. 


\section{References}

1. Abrahamová, D., Hlavacka, F., 2007. Age-related changes of human balance during quiet stance. Physiol Res. 57(6), 957-964.

2. Albertsen, I. M., Ghédira, M., Gracies, J. M., Hutin, É., 2017. Postural stability in young healthy subjects - Impact of reduced base of support, visual deprivation, dual tasking. J Electromyogr Kinesiol. 33, 27-33.

3. Alex, D., Wahi, A., 2014. BSFD: Background subtraction frame difference algorithm for moving object detection and extraction, J Theor Appl Inf Technol. 60, 623-628.

4. Allin, S. J., Beach, C., Mitz, A., Mihailidis, A., 2008. Video based analysis of standing balance in a community center. 30th Annual International Conference of the IEEE Engineering in Medicine and Biology Society, pp. 45314534.

5. Bauer, C., Gröger, I., Rupprecht, R., Gassmann, K. G., 2008. Intrasession reliability of force platform parameters in community-dwelling older adults. Arch Phys Med Rehabil. 89(10), 1977-1982.

6. Ferryman, J., Hogg, D., Sochman, J., Behera, A., Rodriguez-Serranod, J. A., Worgan, S., Li, L., Leung, V., Evans, M., Cornic, P., Herbin, S., Schlenger, S., Dose, M., 2013. Robust abandoned object detection integrating wide area visual surveillance and social context. Pattern Recognition Letters. 34(7), 789-798.

7. Goffredo, M., Schmid, M., Conforto, S., D'Alessio, T., 2006. A markerless sub-pixel motion estimation technique to reconstruct kinematics and estimate the centre of mass in posturography. Med Eng Phys. 28(7), 719-726.

8. Guo, J., Wang, J., Bai, R., Zhang, Y., Li, Y., 2017. A New Moving Object Detection Method Based on Frame - difference and Background Subtraction. International Conference on Industrial Informatics - Computing Technology. Intelligent Technology, Industrial Information Integration. pp. 79-82.

9. Imani, Y., Teyfouri, N., Ahmadzadeh, M. R., Golabbakhsh, M., 2014. A new method for multiple sperm cells tracking. J Med Signals Sens. 4(1), 35-42.

10. Kawa, J., Stępień, P., Kapko, W., Niedziela, A., Derejczyk, J., 2018. Leg movement tracking in automatic videobased one-leg stance evaluation. Comput Med Imaging Graph. 65, 191-199.

11. Lu, G., Zhou, Y., Yu, Y., Du, A., 2011. A novel approach for foreign substances detection in injection using 
clustering and frame difference. Sensors. 11(10), 9121-9135.

12. Piirtola, M., Era, P., 2006. Force platform measurements as predictors of falls among older people - a review. Gerontology. 52(1), 1-16.

13. Pizzigalli, L., Cremasco, M. M., Mulasso, A., Rainoldi, A., 2016. The contribution of postural balance analysis in older adult fallers: A narrative review. J Bodyw Mov Ther. 20 (2), 409-417.

14. Prieto, T. E., Myklebust, J. B., Hoffmann, R. G., Myklebust, B. M., 1996. Measures of postural steadiness: differences between healthy young and elderly adults. IEEE Trans Biomed Eng. 43(9), pp. 956-966.

15. Pua, Y. H., Ong, P. H., Clarck, R. A., Matcher, D. B., Lim, E. C., 2017. Falls efficacy, postural balance, and risk for falls in older adults with falls-related emergency department visits: prospective cohort study. BMC Geriatr. 17(1), 291.

16. Ramya, P., Rajeswari, R., 2016. A Modified frame difference method using correlation coefficient. Procedia Computer Science. 93, 478-485.

17. Singla, N., 2014. Motion Detection Based on Frame Difference Method. International Journal of Information \& Computation Technology. 15, 1559-1565.

18. Wingert, J. R., Welder, C., Foo, P., 2013. Age-related hip proprioception declines: effects on postural sway and dynamic balance. Arch Phys Med Rehabil. 95(2), 253-261.

19. Winter, D. A., Patla, A. E., Ishac, M., Gage, W. H., 2003. Motor mechanisms of balance during quiet standing. J Electromyogr Kinesiol. 13(1), 49-56.

\section{Introduction}

Postural sway is one of the well-known evaluations of postural stability and is not limited to the elderly and patients with balance disorder, but is also used to assess healthy subjects and athletes. The force plates and marker - based systems such as three directional motion analysis systems and accelerometer are generally used in order to gain objective data on postural sway. Since the displacement of center of pressure (COP) during balance tasks reflects balance ability to maintain the center of mass within the base of support (Winter et al., 2003), the COP parameters are used for quantitative evaluation method of postural stability. In particular, the total length of COP displacements (LNG) and the root mean square area (RMS area) are typically used as COP parameters (Albertsen et al., 2017, Bauer et al., 2008). LNG indicates the 
distance of COP, and the RMS area indicates the range of COP. In the preceding study, gradual increase in the COP parameters due to age have been reported (Abrahamová et al., 2007, Prieto et al., 1996, Wingert et al., 2013). Moreover, it has reported that the COP parameters are the variables that distinguish older adult fallers from non-fallers (Pizzigalli et al., 2016), and postural sway might be an indicator of falls risk (Piirtola et al., 2006, Pizzigalli et al., 2016, Pua et al., 2017). Therefore, to understand changes in motor function and the risk of fall caused by aging and diseases with balance disorder objectively, it is necessary to quantify postural stability.

Conversely, there are some disadvantages associated with measurement using equipment such as force plates and marker-based systems. These conventional equipment are expensive, and require much time and technological skill for attaching sensors. Therefore, these measurement equipment are used in limited special environments such as hospitals and laboratories. In most clinical fields, since it is difficult to establish an environment where evaluations of postural stability could be performed using these measurement equipment, it is hard to say that objective evaluations are performed.

The frame subtraction method is one of the key techniques for the automatic image analysis method. The method enables the detection of targeting objects by calculating subtractions between three consecutive images (Alex et al., 2014, Ferryman et al., 2013, Singla, 2014, Guo et al., 2017, Ramya et al., 2016). It has conventionally been applied to count traffic volume on the road and the number of customers in a shop using fixed cameras. As application methods of the frame subtraction method in the medical field, it is used to detect foreign substances in an injection solution (Lu et al., 2011) or to track human sperm cells (Imani et al., 2014). In an environment where nothing moves except for the targeting subject, the motions of the targeting subject can be quantified by the frame subtraction method. By recording each subject during maintenance of single leg standing using one or two digital video cameras, it may be possible to analyze the postural sway, such as motions of the trunk and lower limbs, from the captured images using the frame subtraction method. However, there have been no studies applying the frame subtraction method to evaluation of postural stability. Therefore, we investigate whether this method can be applied to evaluate postural stability during maintenance of single leg standing.

The frame subtraction method is an Open Source Software and included in the OpenCV library (OpenCV 4.1.1). Image analysis by the frame subtraction method only requires standard digital video cameras or digital video cameraequipped tablets on the market, thus the costs could be reduced. Therefore, this system is easier and portable compared to conventional methods, and can be used outside the laboratory. If it is possible to evaluate postural stability using the frame 
subtraction method, this method could be more applicable in the clinical and sports field. However, there has been no attempt to confirm the correlations between the frame subtraction scores using the frame subtraction method and the COP parameters measured using the force plate. Thus, the criterion-related validity of the evaluation of postural stability using the frame subtraction method has not been clarified.

Therefore, the purpose of this study was to verify the criterion-related validity of the evaluation of postural stability using the frame subtraction method.

\section{Methods}

\subsection{Subjects}

The subjects were 22 healthy young people (16 males and 6 females; age, $25.9 \pm 4.6$ years; height, $167.6 \pm 7.7 \mathrm{~cm}$; mass, $60.6 \pm 10.7 \mathrm{~kg}$ ). Those who had a serious history, such as present or past history of ligament injury, fractures, and surgery in the extremities and trunk, were excluded. This study was approved in advance by the ethics committee of Kyoto University (approval number: R1823), and all subjects were given full written explanations before consenting to participate.

\subsection{Motion tasks}

The subjects were asked to keep standing on a single leg for $15 \mathrm{~s}$ with their hands folded across under their chest, and left hip flexed 90 degrees. Although other joints were allowed to move freely, stepping was prohibited. Eyes open conditions and eyes closed conditions were indicated randomly.

\subsection{Collection of data}

The measuring intervals of digital video cameras - equipped tablets and the force plate were synchronized. Within the range of $15 \mathrm{~s}$ of single leg standing, consecutive $10 \mathrm{~s}$ where the subjects remained stable was used for analysis. After several practice trials, these trials were recorded. The data from each trial ware calculated. Mean values from three successful trials were used for statistical analysis. The frame subtraction scores for the same image twice confirmed that the results were in complete agreement. 


\subsubsection{The COP parameters}

To measure the balance ability in the conventional way, the COP parameters during maintenance of single leg standing were collected using a force platform (Kistler Japan Co., Ltd. Tokyo, Japan). The force platform data were sampled at a frequency of $120 \mathrm{~Hz}$ and low-pass-filtered at $10 \mathrm{~Hz}$. LNG, RMS area, range in anterior - posterior direction (AP range), range in medial - lateral direction ML range (ML range) were calculated as the COP parameters.

\subsubsection{The frame subtraction method used in this study}

The motion images were recorded using each digital video camera - equipped tablets from the front and right side of the subjects at a sampling rate of $60 \mathrm{~Hz}$. Fig 1 shows the experimental setup. First, the captured images ware processed by the frame subtraction method (Alex et al., 2014), and the frame subtraction scores on the frontal and the sagittal plane were quantified. Next, the frame subtraction scores on the frontal and the sagittal planes were summed for each frame. The maximum and sum of the frame subtraction scores for $10 \mathrm{~s}$ were calculated as an indicator of the balance ability. In an environment where nothing moves except for the targeting subject, the differences between images are caused by the motions of the subject only. Therefore, the frame subtraction scores obtained by making the differences between images into scores indicate the motions of the subject.

6. $\quad$ Prepare three consecutive images $\left(I_{1}, I_{2}\right.$, and $\left.I_{3}\right)$

7. Calculate the absolute value of the difference between images $I_{1}$ with $I_{2}$, and between images $I_{2}$ with $I_{3}$, and make two different images $\left(I_{d 1}\right.$ and $\left.I_{d 2}\right)$

$$
\begin{aligned}
& I_{d 1}(\mathrm{x}, \mathrm{y})=\left|I_{1}(\mathrm{x}, \mathrm{y})-I_{2}(\mathrm{x}, \mathrm{y})\right| \\
& I_{d 2}(\mathrm{x}, \mathrm{y})=\left|I_{2}(\mathrm{x}, \mathrm{y})-I_{3}(\mathrm{x}, \mathrm{y})\right|
\end{aligned}
$$

8. Calculate the logical product of two subtraction images $\left(I_{d 1}\right.$ and $\left.I_{d 2}\right)$ and create a logical product image $\left(I_{a}\right)$

$$
I_{a}(\mathrm{x}, \mathrm{y})=I_{d 1}(\mathrm{x}, \mathrm{y}) \wedge I_{d 2}(\mathrm{x}, \mathrm{y})
$$

9. Perform binarization processing on logical product image $I a$ ( $T$ is a threshold)

$$
I_{m}(\mathrm{x}, \mathrm{y})=255\left(I_{a}(\mathrm{x}, \mathrm{y})>T\right), 0\left(I_{a}(\mathrm{x}, \mathrm{y}) \leqq T\right)
$$

10. Calculate the total value for each frame and determine it as the frame subtraction score for that frame. 


\subsection{Statistical analysis}

To confirm the criterion-related validity, Spearman's rank correlation coefficient between the frame subtraction scores and the COP parameters was measured. First, correlations between the frame subtraction scores on the frontal plane or sagittal plane with all COP parameters were investigated. The sum of the frame subtraction scores for the frontal and sagittal planes were calculated for each frame. The scores were determined as the frame subtraction score on both planes, and were investigated for correlation with all COP parameters similarly. The level of significance was set at $\mathrm{p}<0.05$. Statistical analysis were performed using IBM SPSS Statistics, version 26 (IBM Japan Ltd., Tokyo, Japan).

\section{Results}

Mean values \pm standard deviation for the frame subtraction scores and the COP parameters were described in Table 1 and Table 2. Table 1 summarizes the results during maintenance of single leg standing with eyes open, and Table 2 summarizes the results during maintenance of single leg standing with eyes closed. Typical examples of the displacement of the COP and the frame subtraction scores on the frontal and sagittal planes in stable and unstable trials were shown in Fig 2. The scatter diagrams of the sum of the frame subtraction scores on the frontal plane and each COP parameter for single leg stance with eyes open (upper panel: a) and closed are shown in Fig 3.

As shown in Table 3, regarding single leg standing with eyes open, there were significant correlations between the maximum of the frame subtraction score on the frontal plane with ML range, as well as between the sum of the frame subtraction score on the frontal plane with LNG, RMS area, AP range, and ML range. As shown in Table 4, regarding single leg standing with eyes closed, there were significant correlations between all frame subtraction scores on the frontal, sagittal, and both planes with all COP parameters, except between the maximum frame subtraction score on the sagittal plane and the ML range.

\section{Discussions}

In this study, the frame subtraction scores were quantified from the captured images of subjects during maintenance of single leg standing recorded using two digital video cameras, and the correlations between the frame subtraction scores and the COP parameters measured using the force plate were confirmed. This study is the first attempt to apply the frame subtraction method to the evaluations of postural stability. The main findings of this study are that there 
were significant positive correlations among the sum of the frame subtraction score on the frontal plane and LNG, RMS area, AP range, and ML range, during maintenance of single leg standing with eyes open and closed.

To evaluate postural stability objectively, it is necessary to quantify the postural sway. In recent years, video analysis technologies have improved tremendously. Previous studies have attempted to apply those techniques to the objective evaluation of postural stability (Allin et al., 2008, Goffredo et al., 2006, Kawa et al., 2018). It has been reported that each body part of subjects during maintenance of stable standing can be estimated from captured images taken with one digital video camera; therefore, the center of mass (COM) trajectories (Goffredo et al., 2006) and the periods of leg up during maintenance of single leg standing (Kawa et al., 2018) were measured. However, in previous studies, validity of the evaluation of postural stability using a conventional marker-less system have not been verified. Therefore, it cannot be said at present that video analysis technologies with marker-less systems have been sufficiently studied in the evaluation of postural stability.

The primary finding of this study is that there are significant positive correlations among the sum of the frame subtraction score on the frontal plane and all COP parameters during maintenance of single leg standing with eyes open and closed. The present results suggest that the sum of frame subtraction score on the frontal plane can indicate instability during maintenance of single leg standing with eyes open and closed. The frame subtraction scores on the frontal and sagittal planes can be measured from captured images taken with digital video cameras from the front and support side easily. Because no measurement equipment or sensors are required, the evaluation of postural stability using the frame subtraction method has low cost, which favors its use in many clinical fields. However, there were differences in results during maintenance of single leg standing between eyes open and closed. Regarding single leg standing with eyes closed, all frame subtraction scores on the frontal, sagittal, and both planes correlated with all COP parameters except between the maximum frame subtraction score on the sagittal plane and the ML range. Regarding single leg standing with eyes open, the sum of the frame subtraction score on the frontal plane only correlated with all COP parameters, and there were no correlations among the frame subtraction scores on the sagittal plane and both planes and all COP parameters. The reason is considered as follows. During maintenance of single leg standing with eyes open, there may have been individual differences in the posture control pattern on the sagittal plane. Although the COP parameters are closely related to trajectories in the center of gravity, joint motions do not necessarily link with trajectories in the center of gravity. 
Sometimes the center of gravity does not move much even with remarkable joint motions such as hip and knee flexions, or the center of gravity move even without remarkable joint motions such as ankle strategy. In the former case, even if the COP parameters are small, the frame subtraction scores, especially on the sagittal plane, would not be small; in the latter case, even if the COP parameters are large, the frame subtraction scores would not be large. For this reason, regarding maintenance of single leg standing with eyes open, the frame subtraction scores on the sagittal plane might have been difficult to relate to the COP parameters. Although the images on the frontal and sagittal planes were analyzed in this study, regardless of single leg standing with eyes open or closed, the frame subtraction scores on the frontal plane only were associated with all COP parameters. Thereby, when evaluating maintenance of single leg standing, it is concluded that the captured images on the frontal plane only are sufficient to analyze the evaluation of postural stability. In other words, it is possible to propose that postural sway during maintenance of single leg standing can be evaluated using one digital video camera only.

Several limitations to this study should be noted. First of all, there are limitations due to the features of the frame subtraction method. Since all moving objects are identified by the frame subtraction method, it necessary to establish one or two digital video cameras and enough space without moving objects except the subject. Another limitation is the accuracy issue. Because the images are filtered for the purpose of cutting noise, the frame subtraction method could not capture inappreciable fluctuations. Thereby, it is thought that the evaluation method of postural stability using the frame subtraction method might not be suitable for easy balance tasks without remarkable joint motions, such as static standing with eyes open in healthy subjects. However, in the evaluation method of postural stability measured using a force plate, the subjects could not be measured accurately unless they stand on the force plate directly, while the evaluation method of postural stability using the frame subtraction method could be applied even under conditions on an unstable board or with weights. Finally, there is a limitation associated with this study. In this study of healthy subjects, the AP ranges during maintenance of single leg standing had significantly positive correlations with the ML ranges $(r=0.63-0.68, \mathrm{p}<0.01)$. Therefore, the frame subtraction scores on the frontal plane, primarily indicating postural sway on the frontal plane, might affect the MP range directly and AP range indirectly. However, it has not yet been verified that the frame subtraction method can be applied to the evaluation of postural stability with balance disorder, especially with remarkable postural sway on the sagittal plane. 
or other subjects.

In conclusion, the current study verified the criterion-related validity of the frame subtraction method based on the evaluation of postural stability. In the balance task with large postural sway, such as single leg standing with eyes closed, except for between the maximum frame subtraction score on the sagittal plane and ML range, all frame subtraction scores on the frontal, sagittal, and both planes were associated with all COP parameters. In the balance task with small postural sway, such as maintenance of single leg standing with eyes open, only the sum of the frame subtraction score on the frontal plane was associated with all COP parameters. In this study, although two digital video cameras were installed ahead and to the right of the subject, regardless of the range of the postural sway, it was possible to measure postural sway with only one digital video camera on the frontal plane, and thus evaluate postural stability. Since the frame subtraction method can reduce costs and time because of its marker-less system, it may be possible to propose a new technique for the evaluation of postural stability during maintenance of single leg standing as an alternative to the use of conventional equipment such as force plates.

\section{Acknowledgements}

We thank all of our study subjects for their participation and engineers of Smart Health Incorporated. This study was supported by a joint research fund from Smart Health Incorporated. 


\section{References}

20. Abrahamová, D., Hlavacka, F., 2007. Age-related changes of human balance during quiet stance. Physiol Res. 57(6), 957-964.

21. Albertsen, I. M., Ghédira, M., Gracies, J. M., Hutin, É., 2017. Postural stability in young healthy subjects - Impact of reduced base of support, visual deprivation, dual tasking. J Electromyogr Kinesiol. 33, 27-33.

22. Alex, D., Wahi, A., 2014. BSFD: Background subtraction frame difference algorithm for moving object detection and extraction, J Theor Appl Inf Technol. 60, 623-628.

23. Allin, S. J., Beach, C., Mitz, A., Mihailidis, A., 2008. Video based analysis of standing balance in a community center. 30th Annual International Conference of the IEEE Engineering in Medicine and Biology Society, pp. 45314534.

24. Bauer, C., Gröger, I., Rupprecht, R., Gassmann, K. G., 2008. Intrasession reliability of force platform parameters in community-dwelling older adults. Arch Phys Med Rehabil. 89(10), 1977-1982.

25. Ferryman, J., Hogg, D., Sochman, J., Behera, A., Rodriguez-Serranod, J. A., Worgan, S., Li, L., Leung, V., Evans, M., Cornic, P., Herbin, S., Schlenger, S., Dose, M., 2013. Robust abandoned object detection integrating wide area visual surveillance and social context. Pattern Recognition Letters. 34(7), 789-798.

26. Goffredo, M., Schmid, M., Conforto, S., D'Alessio, T., 2006. A markerless sub-pixel motion estimation technique to reconstruct kinematics and estimate the centre of mass in posturography. Med Eng Phys. 28(7), 719-726.

27. Guo, J., Wang, J., Bai, R., Zhang, Y., Li, Y., 2017. A New Moving Object Detection Method Based on Frame - difference and Background Subtraction. International Conference on Industrial Informatics - Computing Technology. Intelligent Technology, Industrial Information Integration. pp. 79-82.

28. Imani, Y., Teyfouri, N., Ahmadzadeh, M. R., Golabbakhsh, M., 2014. A new method for multiple sperm cells tracking. J Med Signals Sens. 4(1), 35-42.

29. Kawa, J., Stępień, P., Kapko, W., Niedziela, A., Derejczyk, J., 2018. Leg movement tracking in automatic videobased one-leg stance evaluation. Comput Med Imaging Graph. 65, 191-199.

30. Lu, G., Zhou, Y., Yu, Y., Du, A., 2011. A novel approach for foreign substances detection in injection using 
clustering and frame difference. Sensors. 11(10), 9121-9135.

31. Piirtola, M., Era, P., 2006. Force platform measurements as predictors of falls among older people - a review. Gerontology. 52(1), 1-16.

32. Pizzigalli, L., Cremasco, M. M., Mulasso, A., Rainoldi, A., 2016. The contribution of postural balance analysis in older adult fallers: A narrative review. J Bodyw Mov Ther. 20 (2), 409-417.

33. Prieto, T. E., Myklebust, J. B., Hoffmann, R. G., Myklebust, B. M., 1996. Measures of postural steadiness: differences between healthy young and elderly adults. IEEE Trans Biomed Eng. 43(9), pp. 956-966.

34. Pua, Y. H., Ong, P. H., Clarck, R. A., Matcher, D. B., Lim, E. C., 2017. Falls efficacy, postural balance, and risk for falls in older adults with falls-related emergency department visits: prospective cohort study. BMC Geriatr. 17(1), 291.

35. Ramya, P., Rajeswari, R., 2016. A Modified frame difference method using correlation coefficient. Procedia Computer Science. 93, 478-485.

36. Singla, N., 2014. Motion Detection Based on Frame Difference Method. International Journal of Information \& Computation Technology. 15, 1559-1565.

37. Wingert, J. R., Welder, C., Foo, P., 2013. Age-related hip proprioception declines: effects on postural sway and dynamic balance. Arch Phys Med Rehabil. 95(2), 253-261.

38. Winter, D. A., Patla, A. E., Ishac, M., Gage, W. H., 2003. Motor mechanisms of balance during quiet standing. J Electromyogr Kinesiol. 13(1), 49-56. 


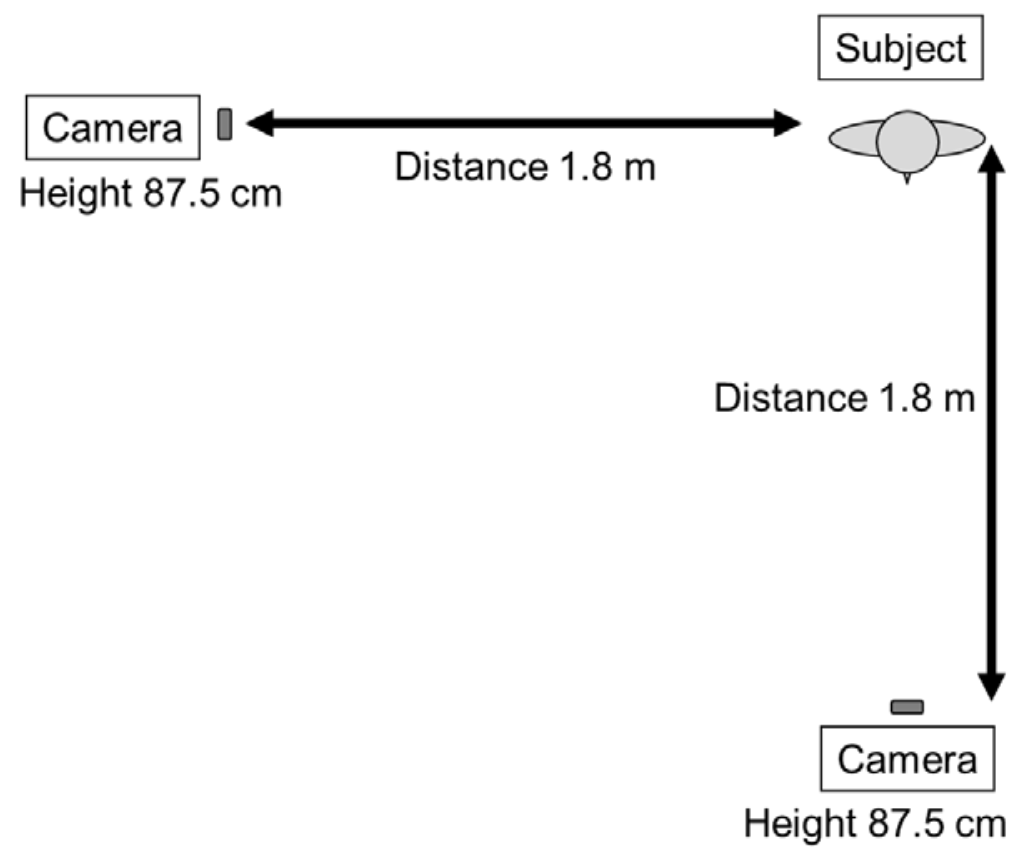

Fig 1. Experimental setup.

The digital video cameras were set 1.8 meters in front and to the right of the subject.

The camera lens height was set to $87.5 \mathrm{~cm}$. 


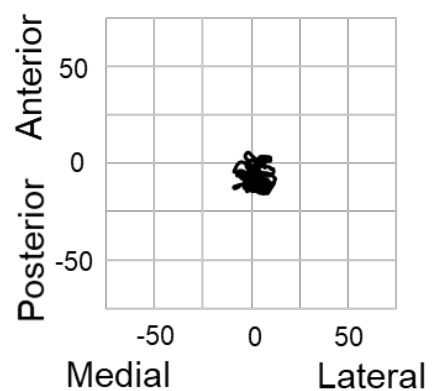

Displacement (mm)

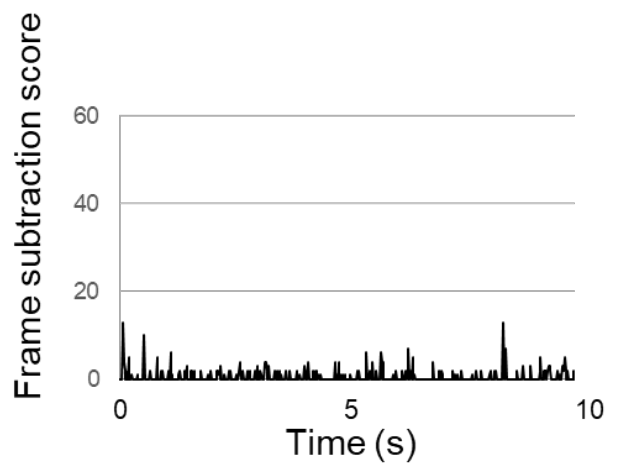

Frontal plane

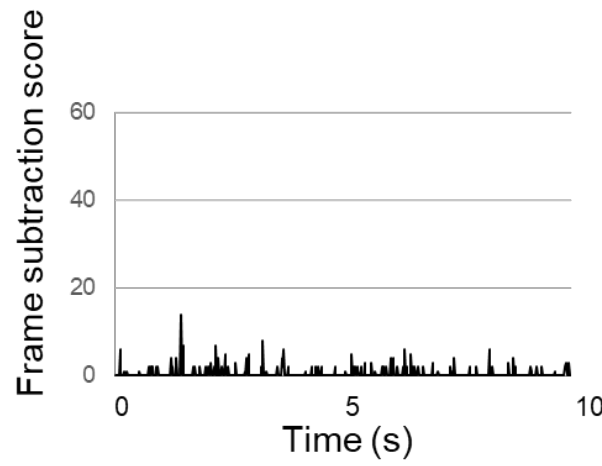

Sagittal plane

a. Typical example in stable trial

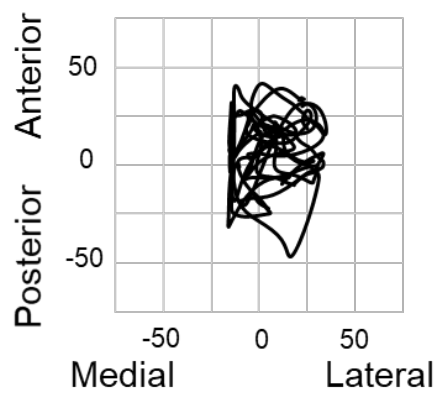

Displacement $(\mathrm{mm})$

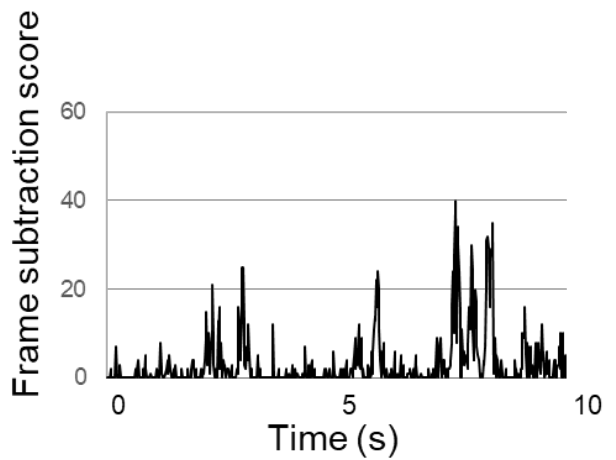

Frontal plane

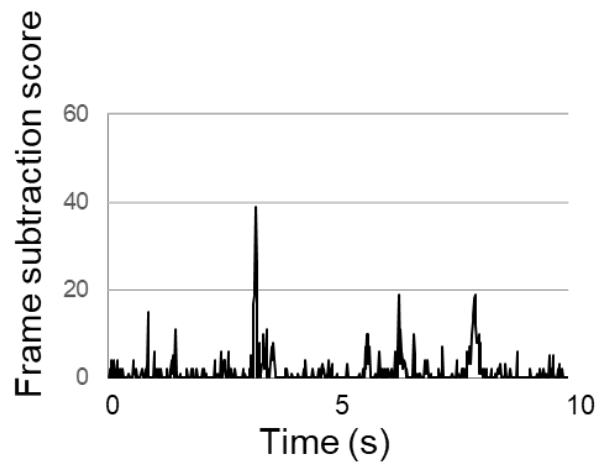

Sagittal plane

b. Typical example in unstable trial

Fig. 2. Typical examples of displacements of the COP and frame subtraction scores on each frontal and sagittal plane, in stable (upper panel: a) and unstable (lower panel: b) trials. 

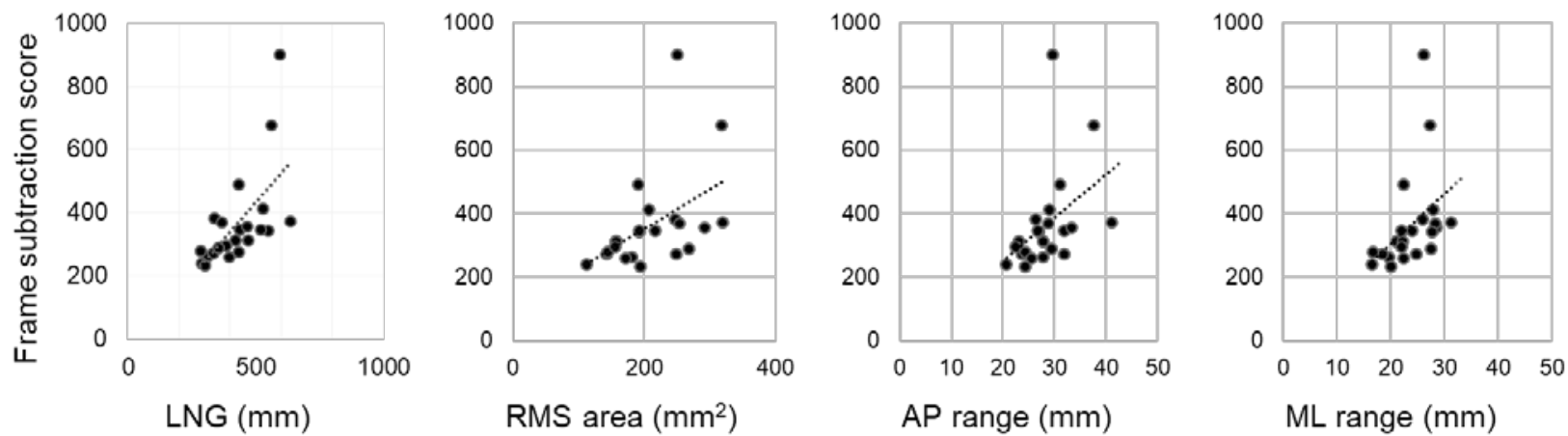

a. Single leg standing with eyes open
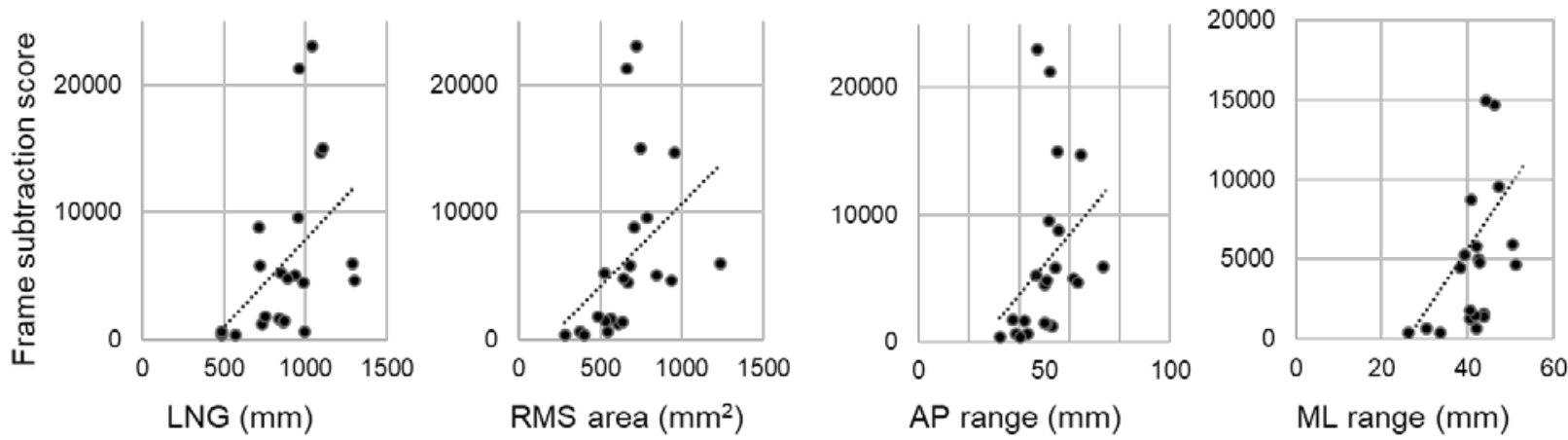

b. Single leg standing with eyes closed

Figure 3. The scatter diagrams of the sum of frame subtraction score on the frontal plane and each COP parameter for single leg standing with eyes open (upper panel: a) and closed (lower panel: b).

Abbreviations: LNG, the total length of COP displacements; RMS area, root mean square area; AP range, range in anterior posterior direction; ML range, range in medial - lateral direction. 
Table 1. Mean values \pm standard deviation for the frame subtraction scores and the COP parameters for single leg standing with eyes open.

\begin{tabular}{|c|c|c|}
\hline Outcome & & Mean value \pm standard deviation \\
\hline \multirow{4}{*}{ COP parameters } & LNG (mm) & $427.48 \pm 102.72$ \\
\hline & RMS area $\left(\mathrm{mm}^{2}\right)$ & $209.24 \pm 58.15$ \\
\hline & AP range (mm) & $28.33 \pm 4.89$ \\
\hline & ML range (mm) & $23.71 \pm 4.08$ \\
\hline \multirow{6}{*}{ Frame subtraction scores } & $\begin{array}{l}\text { Maximum of the frame subtraction score on } \\
\text { the frontal plane }\end{array}$ & $13.38 \pm 4.47$ \\
\hline & $\begin{array}{l}\text { Sum of the frame subtraction score on the } \\
\text { frontal plane }\end{array}$ & $364.29 \pm 154.19$ \\
\hline & $\begin{array}{l}\text { Maximum of the frame subtraction score on } \\
\text { the sagittal plane }\end{array}$ & $42.33 \pm 44.06$ \\
\hline & $\begin{array}{l}\text { Sum of the frame subtraction score on the } \\
\text { sagittal plane }\end{array}$ & $401.71 \pm 170.56$ \\
\hline & $\begin{array}{l}\text { Maximum of the frame subtraction score on } \\
\text { both planes }\end{array}$ & $46.32 \pm 42.53$ \\
\hline & $\begin{array}{l}\text { Sum of the frame subtraction score on both } \\
\text { planes }\end{array}$ & $762.08 \pm 256.20$ \\
\hline
\end{tabular}

Abbreviations: COP; center of pressure, LNG, the total length of COP displacements; RMS area, root mean square area; AP range, range in anterior - posterior direction; ML range, range in medial - lateral direction. 
Table 2. Mean values \pm standard deviation for the frame subtraction scores and the COP parameters for single leg standing with eyes closed.

LNG (mm)

$864.79 \pm 218.76$

RMS area $\left(\mathrm{mm}^{2}\right)$

AP range (mm)

$50.67 \pm 9.69$

ML range (mm)

$41.50 \pm 5.74$

Maximum of the frame subtraction score on

the frontal plane

$121.95 \pm 94.25$

Sum of the frame subtraction score on the

frontal plane

$6253.55 \pm 6644.60$

Maximum of the frame subtraction score on

the sagittal plane

Frame subtraction scores

Sum of the frame subtraction score on the

sagittal plane

$2674.45 \pm 2782.92$

Maximum of the frame subtraction score on

both planes

Sum of the frame subtraction score on both

planes
$188.71 \pm 134.16$

$86.03 \pm 56.96$

$8928.00 \pm 9119.07$

Abbreviations: COP; center of pressure, LNG, the total length of COP displacements; RMS area, root mean square area; AP range, range in anterior - posterior direction; ML range, range in medial - lateral direction. 
Table 3. Spearman's rank correlation coefficient between the frame subtraction score with the COP parameter for single leg standing with eyes open.

LNG RMS area AP range ML range

\begin{tabular}{lcccc}
\hline & $\mathrm{r}=0.35$ & $\mathrm{r}=0.36$ & $\mathrm{r}=0.38$ & $\mathbf{r}=\mathbf{0 . 4 5}$ \\
Maximum frame subtraction score on the frontal plane & $\mathrm{p}=0.11$ & $\mathrm{p}=0.10$ & $\mathrm{p}=0.09$ & $\mathbf{p}<\mathbf{0 . 0 5}$ \\
& $\mathbf{r}=\mathbf{0 . 7 1}$ & $\mathbf{r}=\mathbf{0 . 5 8}$ & $\mathbf{r = 0 . 6 0}$ & $\mathbf{r}=\mathbf{0 . 6 8}$ \\
& $\mathbf{p}<\mathbf{0 . 0 1}$ & $\mathbf{p}<\mathbf{0 . 0 1}$ & $\mathbf{p}<\mathbf{0 . 0 1}$ & $\mathbf{p}<\mathbf{0 . 0 1}$ \\
\hline Sum of the frame subtraction scores on the frontal plane & $\mathrm{r}=-0.17$ & $\mathrm{r}=-0.27$ & $\mathrm{r}=-0.13$ & $\mathrm{r}=-0.31$ \\
& $\mathrm{p}=0.46$ & $\mathrm{p}=0.23$ & $\mathrm{p}=0.56$ & $\mathrm{p}=0.16$ \\
Maximum frame subtraction score on the sagittal plane & $\mathrm{r}=-0.03$ & $\mathrm{r}=-0.11$ & $\mathrm{r}=-0.01$ & $\mathrm{r}=-0.19$ \\
Sum of frame subtraction scores on the sagittal plane & $\mathrm{p}=0.89$ & $\mathrm{p}=0.63$ & $\mathrm{p}=0.96$ & $\mathrm{p}=0.41$ \\
\hline & $\mathrm{r}=-0.21$ & $\mathrm{r}=-0.14$ & $\mathrm{r}=-0.04$ & $\mathrm{r}=-0.20$ \\
Maximum frame subtraction score on both planes & $\mathrm{p}=0.36$ & $\mathrm{p}=0.52$ & $\mathrm{p}=0.87$ & $\mathrm{p}=0.37$ \\
Sum of frame subtraction scores on both planes & $\mathrm{r}=0.39$ & $\mathrm{r}=0.21$ & $\mathrm{r}=0.25$ & $\mathrm{r}=0.26$ \\
& $\mathrm{p}=0.08$ & $\mathrm{p}=0.34$ & $\mathrm{p}=0.26$ & $\mathrm{p}=0.25$ \\
\hline
\end{tabular}

Abbreviations: LNG, the total length of COP displacements; RMS area, root mean square area; AP range, range in anterior posterior direction; ML range, range in medial - lateral direction.

Only statistically significant variables (p values $<0.05$ ) are shown in bold. 
Table 4. Spearman's rank correlation coefficient between the frame subtraction score and the COP parameter for single leg standing with eyes closed.

\begin{tabular}{|c|c|c|c|c|}
\hline & LNG & RMS area & AP range & ML range \\
\hline \multirow{2}{*}{ Maximum frame subtraction score on the frontal plane } & $r=0.52$ & $\mathrm{r}=\mathbf{0 . 7 0}$ & $r=0.61$ & $\mathrm{r}=\mathbf{0 . 4 6}$ \\
\hline & $\mathbf{p}<\mathbf{0 . 0 5}$ & $\mathbf{p}<\mathbf{0 . 0 1}$ & $\mathbf{p}<\mathbf{0 . 0 1}$ & $\mathbf{p}<\mathbf{0 . 0 5}$ \\
\hline \multirow{2}{*}{ Sum of frame subtraction scores on the frontal plane } & $\mathrm{r}=\mathbf{0 . 5 6}$ & $\mathrm{r}=\mathbf{0 . 7 4}$ & $\mathbf{r}=\mathbf{0 . 6 1}$ & $\mathrm{r}=0.53$ \\
\hline & $\mathbf{p}<\mathbf{0 . 0 1}$ & $\mathbf{p}<\mathbf{0 . 0 1}$ & $\mathbf{p}<\mathbf{0 . 0 1}$ & $\mathbf{p}<0.05$ \\
\hline \multirow{2}{*}{ Maximum frame subtraction score on the sagittal plane } & $r=0.43$ & $\mathrm{r}=0.55$ & $r=0.47$ & $\mathrm{r}=0.39$ \\
\hline & $\mathbf{p}<0.05$ & $\mathbf{p}<\mathbf{0 . 0 1}$ & $\mathbf{p}<\mathbf{0 . 0 5}$ & $\mathrm{p}=0.08$ \\
\hline \multirow{2}{*}{ Sum of frame subtraction scores on the sagittal plane } & $\mathrm{r}=\mathbf{0 . 6 1}$ & $\mathrm{r}=\mathbf{0 . 7 1}$ & $\mathrm{r}=0.55$ & $r=0.49$ \\
\hline & $\mathbf{p}<\mathbf{0 . 0 1}$ & $\mathbf{p}<\mathbf{0 . 0 1}$ & $\mathbf{p}<\mathbf{0 . 0 1}$ & $\mathbf{p}<\mathbf{0 . 0 5}$ \\
\hline \multirow{2}{*}{ Maximum frame subtraction score on both planes } & $\mathrm{r}=\mathbf{0 . 5 1}$ & $r=0.66$ & $\mathrm{r}=0.56$ & $\mathrm{r}=\mathbf{0 . 4 8}$ \\
\hline & $\mathbf{p}<\mathbf{0 . 0 5}$ & $\mathbf{p}<\mathbf{0 . 0 1}$ & $\mathbf{p}<\mathbf{0 . 0 1}$ & $\mathbf{p}<\mathbf{0 . 0 5}$ \\
\hline \multirow{2}{*}{ Sum of the frame subtraction score on the both planes } & $r=0.60$ & $\mathbf{r}=\mathbf{0 . 7 7}$ & $r=0.64$ & $r=0.57$ \\
\hline & $\mathbf{p}<\mathbf{0 . 0 1}$ & $\mathbf{p}<\mathbf{0 . 0 1}$ & $\mathbf{p}<\mathbf{0 . 0 1}$ & $\mathbf{p}<\mathbf{0 . 0 1}$ \\
\hline
\end{tabular}

Abbreviations: LNG, the total length of COP displacements; RMS area, root mean square area; AP range, range in anterior posterior direction; ML range, range in medial - lateral direction.

Only statistically significant variables ( $\mathrm{p}$ values $<0.05$ ) are shown in bold. 